Pacific

Journal of

Mathematics

ON A QUOTIENT OF THE UNRAMIFIED IWASAWA MODULE OVER AN ABELIAN NUMBER FIELD, II

HUMio ICHIMURA 


\title{
ON A QUOTIENT OF THE UNRAMIFIED IWASAWA MODULE OVER AN ABELIAN NUMBER FIELD, II
}

\author{
Humio ICHIMURA
}

Let $p$ be an odd prime number, $k$ an imaginary abelian field containing a primitive $p$-th root of unity, and $k_{\infty} / k$ the cyclotomic $\mathrm{Z}_{p}$-extension. Denote by $L / k_{\infty}$ the maximal unramified pro- $p$ abelian extension, and by $L^{\prime}$ the maximal intermediate field of $L / k_{\infty}$ in which all prime divisors of $k_{\infty}$ over $p$ split completely. Let $N / k_{\infty}\left(\operatorname{resp} . N^{\prime} / k_{\infty}\right)$ be the pro- $p$ abelian extension generated by all $p$-power roots of all units (resp. $p$-units) of $k_{\infty}$. In the previous paper, we proved that the $\mathbf{Z}_{p}$-torsion subgroup of the odd part of the Galois group $\operatorname{Gal}\left(N \cap L / k_{\infty}\right)$ is isomorphic, over the group ring $\mathrm{Z}_{p}[\mathrm{Gal}(k / \mathrm{Q})]$, to a certain standard subquotient of the even part of the ideal class group of $k_{\infty}$. In this paper, we prove that the same holds also for the Galois group $\operatorname{Gal}\left(N^{\prime} \cap L^{\prime} / k_{\infty}\right)$.

\section{Introduction.}

Let $p$ be a fixed odd prime number, $k$ an imaginary abelian field containing a primitive $p$-th root $\zeta_{p}$ of unity, and $k_{\infty} / k$ the cyclotomic $\mathbf{Z}_{p}$-extension. Let $L / k_{\infty}$ be the maximal unramified pro- $p$ abelian extension, and $L^{\prime}$ the maximal intermediate field of $L / k_{\infty}$ in which all prime divisors of $k_{\infty}$ over $p$ split completely. We put

$$
N=k_{\infty}\left(\epsilon^{1 / p^{n}} \mid \epsilon \in E_{\infty}, n \geq 1\right), \quad N^{\prime}=k_{\infty}\left(\epsilon^{1 / p^{n}} \mid \epsilon \in E_{\infty}^{\prime}, n \geq 1\right),
$$

where $E_{\infty}$ (resp. $E_{\infty}^{\prime}$ ) is the group of units (resp. p-units) of $k_{\infty}$. Put

$$
\begin{aligned}
\mathcal{X}=\operatorname{Gal}\left(L / k_{\infty}\right), & \mathcal{Y}=\operatorname{Gal}\left(N \cap L / k_{\infty}\right), \\
\mathcal{X}^{\prime}=\operatorname{Gal}\left(L^{\prime} / k_{\infty}\right), & \mathcal{Y}^{\prime}=\operatorname{Gal}\left(N^{\prime} \cap L^{\prime} / k_{\infty}\right),
\end{aligned}
$$

and let $\mathcal{X}^{-}, \mathcal{Y}^{-}, \mathcal{X}^{\prime-}, \mathcal{Y}^{\prime-}$ be the odd parts of the respective Galois groups. It is well-known that $\mathcal{X}^{-}$is (finitely generated and) torsion free over $\mathbf{Z}_{p}$ (cf. Washington [14, Corollary 13.29]). It is also known (and is shown similarly) that $\mathcal{X}^{\prime-}$ is torsion free over $\mathbf{Z}_{p}$. One naturally asks whether or not the quotients $\mathcal{Y}^{-}$of $\mathcal{X}^{-}$and $\mathcal{Y}^{\prime-}$ of $\mathcal{X}^{\prime-}$ are also torsion free over $\mathbf{Z}_{p}$. This question arised in the previous investigation [5], [6] on a power integral basis problem over cyclotomic $\mathbf{Z}_{p}$-extensions.

Let $A_{\infty}$ be the ideal class group of $k_{\infty}$, and $A_{\infty}^{+}$its even part. It is conjectured by Greenberg [4] that $A_{\infty}^{+}=\{0\}$, which is far from being settled 
in general. Under this conjecture, it is known that $\mathcal{Y}^{-}=\mathcal{X}^{-}$and $\mathcal{Y}^{\prime-}=\mathcal{X}^{\prime-}$, and hence $\mathcal{Y}^{-}$and $\mathcal{Y}^{\prime-}$ are torsion free over $\mathbf{Z}_{p}$.

In the preceding paper [7], we proved that the $\mathbf{Z}_{p}$-torsion subgroup Tor $\mathcal{Y}^{-}$ of $\mathcal{Y}^{-}$is isomorphic, over the group $\operatorname{ring} \mathbf{Z}_{p}[\operatorname{Gal}(k / \mathbf{Q})]$, to a certain standard subquotient of $A_{\infty}^{+}$(under the assumption that $p$ does not divide the degree $[k: \mathbf{Q}])$. Further, we gave some assertions on the vanishing of this subquotient.

Let $\mathcal{O}_{\infty}$ be the ring of integers of $k_{\infty}$, and $\mathcal{O}_{\infty}^{\prime}=\mathcal{O}_{\infty}[1 / p]$ the ring of $p$-integers. The pairs $(L, N)$ and $\left(L^{\prime}, N^{\prime}\right)$ are objects associated to $\mathcal{O}_{\infty}$ and $\mathcal{O}_{\infty}^{\prime}$, respectively. Since $k_{\infty} / k$ is wildly ramified at $p$, it is often more natural to use the $p$-integers $\mathcal{O}_{\infty}^{\prime}$ than $\mathcal{O}_{\infty}$. Therefore, it is desirable to obtain a corresponding result for the pair $\left(\mathcal{X}^{\prime}, \mathcal{Y}^{\prime}\right)$. In this paper, we prove that the $\mathbf{Z}_{p}$-torsion subgroup Tor $\mathcal{Y}^{\prime-}$ of $\mathcal{Y}^{\prime-}$ is also isomorphic to the above mentioned subquotient of $A_{\infty}^{+}$as a $\mathbf{Z}_{p}[\operatorname{Gal}(k / \mathbf{Q})]$-module. Namely, Tor $\mathcal{Y}^{-}$and Tor $\mathcal{Y}^{\prime-}$ are isomorphic to each other over $\mathbf{Z}_{p}[\operatorname{Gal}(k / \mathbf{Q})]$.

\section{Results.}

Let $k$ be an imaginary abelian field with $\zeta_{p} \in k^{\times}$, and $\Delta=\operatorname{Gal}(k / \mathbf{Q})$, $\Gamma=\operatorname{Gal}\left(k_{\infty} / k\right)$. We assume that

$$
p \text { does not divide the degree }[k: \mathbf{Q}] \text {. }
$$

Then, we have a canonical decomposition

$$
\operatorname{Gal}\left(k_{\infty} / \mathbf{Q}\right)=\Delta \times \Gamma .
$$

A $\mathbf{Q}_{p}$-valued character of $\Delta$ defined and irreducible over $\mathbf{Q}_{p}$ is simply called a $\mathbf{Q}_{p}$-character. For a $\mathbf{Q}_{p}$-character $\Phi$ of $\Delta$ and a $\mathbf{Z}_{p}[\Delta]$-module $X$, we denote by $X^{+}, X^{-}$and $X(\Phi)$ the even part, the odd part and the $\Phi$-component $e_{\Phi} X$ of $X$, respectively. Here, $e_{\Phi}$ is the idempotent of $\mathbf{Q}_{p}[\Delta]$ defined by

$$
e_{\Phi}=\frac{1}{|\Delta|} \sum_{\sigma \in \Delta} \Phi(\sigma) \sigma^{-1},
$$

which is an element of $\mathbf{Z}_{p}[\Delta]$ by the assumption $(\mathrm{H})$.

Throughout this paper, we fix an even $\mathbf{Q}_{p}$-character $\Psi$ of $\Delta$ and its irreducible component $\psi$ over the algebraic closure $\overline{\mathbf{Q}}_{p}$. Denote by $\Psi^{*}$ and $\psi^{*}$ the odd characters of $\Delta$ associated to $\Psi$ and $\psi$ by

$$
\Psi^{*}(\sigma)=\omega(\sigma) \Psi\left(\sigma^{-1}\right), \quad \psi^{*}(\sigma)=\omega(\sigma) \psi\left(\sigma^{-1}\right), \quad(\sigma \in \Delta),
$$

respectively, where $\omega$ is the character of $\Delta$ representing the Galois action on $\zeta_{p}$. We often regard $\psi$ and $\psi^{*}$ as primitive Dirichlet characters.

Let $k_{n}(n \geq 0)$ be the $n$-th layer of $k_{\infty} / k$ with $k_{0}=k$, and $A_{n}$ the Sylow $p$-subgroup of the ideal class group of $k_{n}$. Let

$$
A_{\infty}=\underset{\lim }{\longrightarrow} A_{n}
$$


be the inductive limit with respect to the inclusion maps $k_{n} \rightarrow k_{m}(n<m)$. Denote by $\widetilde{A}_{0}$ the image of $A_{0}$ in $A_{\infty}$. Let $A_{\infty}^{\Gamma}$ be the elements of $A_{\infty}$ fixed by the action of $\Gamma=\operatorname{Gal}\left(k_{\infty} / k\right)$. It is known (cf. [4, Proposition 1]) that $\left(A_{\infty}^{\Gamma}\right)^{+}$is a finite abelian group as a consequence of the Leopoldt conjecture for $(k, p)$ proved by Brumer $[\mathbf{1}]$. Hence, so is $\left(A_{\infty}^{\Gamma} / \widetilde{A}_{0}\right)(\Psi)$. On the other hand, Tor $\mathcal{Y}\left(\Psi^{*}\right)$ and $\operatorname{Tor} \mathcal{Y}^{\prime}\left(\Psi^{*}\right)$ are also finite since $\mathcal{X}^{-}$is finitely generated over $\mathbf{Z}_{p}$ by the theorem of Ferrero and Washington [2]. For the trivial character $\Psi_{0}$, it is known (cf. [14, Proposition 6.16]) that $A_{\infty}\left(\Psi_{0}\right)=\{0\}$ and $\mathcal{X}\left(\Psi_{0}^{*}\right)=\{0\}$. So, in what follows, we assume that $\Psi$ is nontrivial (and even).

In [7], we proved the following:

Theorem 1. The finite abelian groups $\operatorname{Tor} \mathcal{Y}\left(\Psi^{*}\right)$ and $\left(A_{\infty}^{\Gamma} / \widetilde{A}_{0}\right)(\Psi)$ are isomorphic to each other.

As for the subquotient $A_{\infty}^{\Gamma} / \widetilde{A}_{0}$ of $A_{\infty}$, we proved in [7, Proposition 1] the following:

Proposition 1. When $\psi(p) \neq 1$, we have $\left(A_{\infty}^{\Gamma} / \widetilde{A}_{0}\right)(\Psi)=\{0\}$.

For more on this subquotient, see [7, Proposition 3] and [8].

The main result of this paper is as follows.

Theorem 2. Tor $\mathcal{Y}^{\prime}\left(\Psi^{*}\right)$ is isomorphic to $\left(A_{\infty}^{\Gamma} / \widetilde{A}_{0}\right)(\Psi)$ as an abelian group.

We obtain the following corollary from Theorems 1 and 2 .

Corollary. The $\mathbf{Z}_{p}[\Delta]$-modules Tor $\mathcal{Y}^{-}$and Tor $\mathcal{Y}^{-}$are isomorphic to each other.

We put

$$
\mathcal{H}=\operatorname{Gal}\left(N / k_{\infty}\right) \quad \text { and } \quad \mathcal{H}^{\prime}=\operatorname{Gal}\left(N^{\prime} / k_{\infty}\right) .
$$

It is known (cf. [6, Claim (page 97)]) that, by the restriction map,

$$
\mathcal{H}^{\prime}\left(\Psi^{*}\right)=\mathcal{H}\left(\Psi^{*}\right)
$$

This is because the Leopoldt conjecture for $\left(k_{n}, p\right)$ holds for all $n \geq 0$ by [1] It is also known (see Section 4.2 (Proof of Lemma 1)) that, by the restriction map,

$$
\mathcal{X}\left(\Psi^{*}\right)=\mathcal{X}^{\prime}\left(\Psi^{*}\right) \quad \text { when } \psi^{*}(p) \neq 1 .
$$

Therefore, when $\psi^{*}(p) \neq 1$, we have $\mathcal{Y}^{\prime}\left(\Psi^{*}\right)=\mathcal{Y}\left(\Psi^{*}\right)$. By this and Proposition 1, we see that Theorem 2 follows immediately from Theorem 1 and the following:

Theorem 3. When $\psi^{*}(p)=1, \mathcal{Y}^{\prime}\left(\Psi^{*}\right)$ is torsion free over $\mathbf{Z}_{p}$. 
Remark 1. Let $A_{n}^{\prime}$ be the Sylow $p$-subgroup of the $p$-ideal-class group of $k_{n}$ in the sense of Iwasawa [10, Section 4.3], and let $A_{\infty}^{\prime}$ be the inductive limit of $A_{n}^{\prime}$ with respect to the inclusion maps $k_{n} \rightarrow k_{m}(n<m)$. Denote by $\widetilde{A}_{0}^{\prime}$ the image of $A_{0}^{\prime}$ in $A_{\infty}^{\prime}$. To talk about the Galois groups $\mathcal{X}^{\prime}, \mathcal{Y}^{\prime}$, it is more natural to use $A_{\infty}^{\prime}$ than $A_{\infty}$. However, it is known (cf. [4, Corollary]) that the natural projections

$$
A_{\infty}^{+} \longrightarrow A_{\infty}^{\prime+} \quad \text { and } \quad \widetilde{A}_{0}^{+} \longrightarrow \widetilde{A}_{0}^{\prime+}
$$

are isomorphisms as a consequence of the Leopoldt conjecture for $\left(k_{n}, p\right)$ $(n \geq 0)$.

Remark 2. It is conjectured that $A_{\infty}^{+}=\{0\}$ (cf. [4]). We have many numerical examples of $(k, p)$ with $A_{\infty}^{+}=\{0\}$, but no counter examples (see Kraft and Schoof [11], Kurihara [12], Sumida and the author [9]). However, the conjecture is not yet proved to be true in general.

\section{Proof of Theorem 3.}

We recall a standard notation. Let $O=O_{\psi}$ be the subring of $\overline{\mathbf{Q}}_{p}$ generated by the values of $\psi$ over $\mathbf{Z}_{p}$. We identify the subring $e_{\Psi^{*}} \mathbf{Z}_{p}[\Delta]$ of $\mathbf{Z}_{p}[\Delta]$ with $O$ by sending $e_{\Psi^{*}} \sigma$ to $\psi^{*}(\sigma),(\sigma \in \Delta)$. Then, for a $\mathbf{Z}_{p}[\Delta]$-module $X, X\left(\Psi^{*}\right)$ is regarded as an $O$-module. We fix a topological generator $\gamma$ of $\Gamma$. We identify, as usual, the completed group ring $e_{\Psi^{*}} \mathbf{Z}_{p}[\Delta][[\Gamma]]$ with the power series ring $\Lambda=O[[T]]$ by $\gamma=1+T$ and the above identification. Thus, for a $\mathbf{Z}_{p}[\Delta][[\Gamma]]$-module $X$ (such as several Galois groups over $k_{\infty}$ ), we can regard $X\left(\Psi^{*}\right)$ as a module over $O$ or $\Lambda$. We denote by $q$ the element of $p \mathbf{Z}_{p}$ such that $\zeta^{\gamma}=\zeta^{1+q}$ for all $\zeta \in \mu_{p^{\infty}}$.

Let $M / k_{\infty}$ be the maximal pro- $p$ abelian extension unramified outside $p$. The fields $N, L, N^{\prime}$ and $L^{\prime}$ are intermediate fields of $M / k_{\infty}$. We put

$$
\begin{aligned}
\mathcal{G}=\operatorname{Gal}\left(M / k_{\infty}\right), & \mathcal{Z}^{\prime}=\operatorname{Gal}\left(M / N^{\prime}\right) \\
\mathcal{I}=\operatorname{Gal}(M / L), & \mathcal{I}^{\prime}=\operatorname{Gal}\left(M / L^{\prime}\right) .
\end{aligned}
$$

For a $\mathbf{Q}_{p}$-character $\Phi$ of $\Delta$, denote by $M(\Phi)$ the intermediate field of $M / k_{\infty}$ corresponding to $\bigoplus_{\Phi^{\prime}}^{\prime} \mathcal{G}\left(\Phi^{\prime}\right)$ by Galois theory where $\Phi^{\prime}$ runs over the $\mathbf{Q}_{p^{-}}$ characters of $\Delta$ with $\Phi^{\prime} \neq \Phi$. Then, $\operatorname{Gal}\left(M(\Phi) / k_{\infty}\right)=\mathcal{G}(\Phi)$. We define $N(\Phi), L(\Phi)$, etc, in a similar way.

As we have mentioned in Section $2, \mathcal{H}^{\prime}\left(\Psi^{*}\right)=\mathcal{H}\left(\Psi^{*}\right)$. Therefore, by the assertion [6, Lemma 1] on $\mathcal{H}\left(\Psi^{*}\right)$, there exists an injective $\Lambda$-homomorphism

$$
\iota: \mathcal{H}^{\prime}\left(\Psi^{*}\right) \hookrightarrow \begin{cases}\Lambda, & \text { when } \psi(p) \neq 1, \\ \Lambda \oplus \Lambda /(T-q), & \text { when } \psi(p)=1,\end{cases}
$$

with a finite cokernel. This is the $\Delta$-decomposed version of $[\mathbf{1 0}$, Theorem 15]. In the next section, we prove the following two lemmas. 
Lemma 1. There exists a $\Lambda$-isomorphism:

$$
\mathcal{I}^{\prime}\left(\Psi^{*}\right) \cong \begin{cases}\Lambda, & \text { when } \psi(p) \neq 1, \\ \Lambda \oplus \Lambda /(T-q), & \text { when } \psi(p)=1 .\end{cases}
$$

Lemma 2. We have $M\left(\Psi^{*}\right)=N^{\prime}\left(\Psi^{*}\right) L^{\prime}\left(\Psi^{*}\right)$.

Proof of Theorem 3. Assume that $\psi^{*}(p)=1$. We put

$$
\overline{\mathcal{I}^{\prime}}\left(\Psi^{*}\right)=\mathcal{I}^{\prime}\left(\Psi^{*}\right) \mathcal{Z}^{\prime}\left(\Psi^{*}\right) / \mathcal{Z}^{\prime}\left(\Psi^{*}\right) .
$$

Then, we have $\overline{\mathcal{I}^{\prime}}\left(\Psi^{*}\right) \subseteq \mathcal{H}^{\prime}\left(\Psi^{*}\right)$, and

$$
\mathcal{Y}^{\prime}\left(\Psi^{*}\right) \cong \mathcal{H}^{\prime}\left(\Psi^{*}\right) / \overline{\mathcal{I}^{\prime}}\left(\Psi^{*}\right) .
$$

As $\psi^{*}(p)=1$, we see from Lemmas 1 and 2 that

$$
\overline{\mathcal{I}^{\prime}}\left(\Psi^{*}\right) \cong \mathcal{I}^{\prime}\left(\Psi^{*}\right) \cong \Lambda \text {. }
$$

Let $\iota$ be an embedding of $\mathcal{H}^{\prime}\left(\Psi^{*}\right)$ into $\Lambda$ with a finite cokernel. By the above, the image $\iota\left(\overline{\mathcal{I}^{\prime}}\left(\Psi^{*}\right)\right)$ of $\overline{\mathcal{I}^{\prime}}\left(\Psi^{*}\right)$ equals a principal ideal $(f)$ of $\Lambda$ for some $f \in \Lambda$. Therefore, we obtain an injective $\Lambda$-homomorphism

$$
\mathcal{Y}^{\prime}\left(\Psi^{*}\right) \hookrightarrow \Lambda /(f)
$$

with a finite cokernel. On the other hand, $f$ is relatively prime to $p$ by [2]. Hence, $\mathcal{Y}^{\prime}\left(\Psi^{*}\right)$ is torsion free over $\mathbf{Z}_{p}$.

\section{Proof of lemmas.}

4.1. Preliminaries. In this subsection, we give and recall some assertions on some groups of local universal norms of $k_{\infty} / k$ and the Galois groups $\mathcal{I}=\operatorname{Gal}(M / L), \mathcal{I}^{\prime}=\operatorname{Gal}\left(M / L^{\prime}\right)$. For a while, we fix a prime ideal $\mathfrak{p}$ of $k$ over $p$. We denote the unique prime ideal of $k_{n}$ over $\mathfrak{p}$ simply by $\mathfrak{p}$. Let $k_{n, \mathfrak{p}}$ be the completion of $k_{n}$ at $\mathfrak{p}$, and $\mathcal{U}_{n, \mathfrak{p}}$ the group of principal units of $k_{n, \mathfrak{p}}$. Let

$$
\mathcal{V}_{n, \mathfrak{p}}=\bigcap_{m \geq n} N_{m / n} \mathcal{U}_{m, \mathfrak{p}} \quad \text { and } \quad \mathcal{W}_{n, \mathfrak{p}}=\bigcap_{m \geq n} N_{m / n}\left(\left(k_{m, \mathfrak{p}}^{\times}\right)^{(p)}\right)
$$

be the groups of universal norms. Here, $N_{m / n}$ denotes the norm map from $k_{m}^{\times}$to $k_{n}^{\times}$, and for an abelian group $X, X^{(p)}$ denotes the maximal pro- $p$ quotient. We put

$$
\mathcal{U}_{n}=\prod_{\mathfrak{p} \mid p} \mathcal{U}_{n, \mathfrak{p}}, \quad \mathcal{V}_{n}=\prod_{\mathfrak{p} \mid p} \mathcal{V}_{n, \mathfrak{p}}, \quad \mathcal{W}_{n}=\prod_{\mathfrak{p} \mid p} \mathcal{W}_{n, \mathfrak{p}}
$$

where $\mathfrak{p}$ runs over the primes of $k$ over $p$. These are closed subgroups of the maximal pro- $p$ quotient $\widehat{k_{n}^{\times}}=\left(\prod_{\mathfrak{p} \mid p} k_{n, \mathfrak{p}}^{\times}\right)^{(p)}$. Denote by $\varphi_{n}$ the natural embedding of $k_{n}^{\times}$into $\widehat{k_{n}^{\times}}$. Let $E_{n}$ (resp. $E_{n}^{\prime}$ ) be the group of units (resp. $p$ units) of $k_{n}$, and let $\mathcal{E}_{n}$ (resp. $\left.\mathcal{E}_{n}^{\prime}\right)$ be the closure of $\varphi_{n}\left(E_{n}\right)\left(\right.$ resp. $\varphi_{n}\left(E_{n}^{\prime}\right)$ ) 
in $\widehat{k_{n}^{\times}}$. Let $\mathcal{U}_{\infty}, \mathcal{E}_{\infty}, \mathcal{W}_{\infty}, \mathcal{E}_{\infty}^{\prime}$ be the projective limits of $\mathcal{U}_{n}, \mathcal{E}_{n}, \mathcal{W}_{n}, \mathcal{E}_{n}^{\prime}$ with respect to the relative norms, respectively:

$$
\mathcal{U}_{\infty}=\lim _{\longleftarrow} \mathcal{U}_{n}\left(=\lim _{\longleftarrow} \mathcal{V}_{n}\right), \quad \mathcal{E}_{\infty}^{\prime}=\lim _{\longleftarrow} \mathcal{E}_{n}^{\prime}\left(=\lim _{\longleftarrow}\left(\mathcal{W}_{n} \cap \mathcal{E}_{n}^{\prime}\right)\right), \quad \text { etc. }
$$

These groups are naturally regarded as modules over $\mathbf{Z}_{p}[\Delta][[\Gamma]]$.

Lemma 3. The projection $P: \mathcal{W}_{\infty} \rightarrow \mathcal{W}_{0}$ induces an isomorphism

$$
\mathcal{W}_{\infty} / \mathcal{W}_{\infty}^{T} \cong \mathcal{W}_{0}
$$

Proof. It is clear that the projection $P$ is surjective and that $\mathcal{W}_{\infty}^{T} \subseteq \operatorname{ker} P$. So, it suffices to show that $\operatorname{ker} P \subseteq \mathcal{W}_{\infty}^{T}$. Let $u=\left(u_{n}\right)_{n \geq 0}$ be an element of ker $P$ with $u_{n} \in \mathcal{W}_{n}$. As $u_{0}=1$, we see that $u_{n}$ is contained in $\mathcal{U}_{n}$. We can write $u_{n}=w_{n}^{T}$ for some $w_{n} \in \prod_{\mathfrak{p} \mid p} k_{n, \mathfrak{p}}^{\times}$by Hilbert Satz 90. Hence, $u_{n}=\bar{w}_{n}^{T}, \bar{w}_{n}$ being the projection of $w_{n}$ in $\widehat{k_{n}^{\times}}$. Denote by $x^{(n)}$ the element of the product $X=\prod_{\ell} \widehat{k_{\ell}^{\times}}$whose $\ell$-th component is $N_{n / \ell}\left(\bar{w}_{n}\right)$ (resp. 1) for $\ell \leq n$ (resp. $\ell>n)$. Since $X$ is compact, $\left\{x^{(n)}\right\}$ has an accumulation point $x$ in $X$. We easily see that $x \in \mathcal{W}_{\infty}$ and $x^{T}=u$. Therefore, ker $P \subseteq \mathcal{W}_{\infty}^{T}$.

By class field theory, it is known (cf. [14, Corollary 13.6]) that the inertia group $\mathcal{I}$ is canonically isomorphic to $\mathcal{U}_{\infty} / \mathcal{E}_{\infty}$ over $\mathbf{Z}_{p}[\Delta][[\Gamma]]$. As $\Psi^{*}$ is odd and $\Psi^{*} \neq \omega$, it follows that $\mathcal{E}_{\infty}\left(\Psi^{*}\right)=\{0\}$ by a theorem on units of CM-fields (cf. [14, Theorem 4.12]). Therefore, we obtain a $\Lambda$-isomorphism

$$
\mathcal{I}\left(\Psi^{*}\right) \cong \mathcal{U}_{\infty}\left(\Psi^{*}\right) .
$$

On the $\Lambda$-structure of $\mathcal{U}_{\infty}$, it is known (cf. Gillard [3, Proposition 1]) that

$$
\mathcal{U}_{\infty}\left(\Psi^{*}\right) \cong \begin{cases}\Lambda, & \text { when } \psi(p) \neq 1 \\ \Lambda \oplus \Lambda /(T-q), & \text { when } \psi(p)=1 .\end{cases}
$$

It is also known (cf. [3, Proposition 2]) that

$$
\mathcal{V}_{0}\left(\Psi^{*}\right) \cong \begin{cases}O, & \text { when } \psi(p) \neq 1 \text { and } \psi^{*}(p) \neq 1, \\ O \oplus O / q, & \text { when } \psi(p)=1, \\ \{0\}, & \text { when } \psi^{*}(p)=1 .\end{cases}
$$

As for the decomposition group $\mathcal{I}^{\prime}$, we need to prove the following:

Proposition 2. The reciprocity law map induces a canonical isomorphism

$$
\mathcal{I}^{\prime} \cong \mathcal{W}_{\infty} / \mathcal{E}_{\infty}^{\prime}
$$

over $\mathbf{Z}_{p}[\Delta][[\Gamma]]$.

Proof. Let $M_{n}$ (resp. $L_{n}^{\prime}$ ) be the maximal abelian extension of $k_{n}$ contained in $M$ (resp. $L^{\prime}$ ). It suffices to prove that

$$
\operatorname{Gal}\left(M_{n} / L_{n}^{\prime}\right) \cong \mathcal{W}_{n} /\left(\mathcal{W}_{n} \cap \mathcal{E}_{n}^{\prime}\right)
$$


since $\mathcal{I}^{\prime}$ is the projective limit of $\operatorname{Gal}\left(M_{n} / L_{n}^{\prime}\right)$ with respect to the restriction maps. It suffices to show the assertion (6) only when $n=0$ by considering $k_{n}$ as the base field.

For an integer $m(\geq 0)$, we put

$$
W^{(m)}=\prod_{\mathfrak{p} \mid p} N_{m / 0} k_{m, \mathfrak{p}}^{\times}\left(\supseteq \mathcal{U}_{0}^{p^{m}}\right) .
$$

For a prime divisor $\mathfrak{q}$ of $k$ relatively prime to $p$, let $U_{\mathfrak{q}}$ be the group of local units (resp. the multiplicative group) of the completion $k_{\mathfrak{q}}$ of $k$ at $\mathfrak{q}$ when $\mathfrak{q}$ is finite (resp. infinite). Let $J_{k}$ be the group of idèles of $k$. We define its subgroups $A, B, C$ as follows:

$$
A=W^{(m)} \times \prod_{\mathfrak{q} \nmid p}\{1\}, \quad B=\mathcal{U}_{0}^{p^{m}} \times \prod_{\mathfrak{q} \nmid p}\{1\}, \quad C=\prod_{\mathfrak{p} \mid p}\{1\} \times \prod_{\mathfrak{q} \nmid p} U_{\mathfrak{q}},
$$

where $\mathfrak{p}$ (resp. $\mathfrak{q}$ ) runs over the primes of $k$ dividing $p$ (resp. relatively prime to $p$ ).

Denote by $H$ the Hilbert $p$-class field of $k$. Let $M_{0, m}$ be the maximal intermediate field of $M_{0} / H$ whose Galois group over $H$ is of exponent $p^{m}$. Clearly, $M_{0, m}$ contains $k_{m}$. Let $L_{0, m}^{\prime}$ be the maximal intermediate field of $M_{0, m} / k_{m}$ in which all prime divisors of $k_{m}$ over $p$ split completely. We have a natural isomorphism

$$
\operatorname{Gal}\left(M_{0} / L_{0}^{\prime}\right) \cong \lim \operatorname{Gal}\left(M_{0, m} / L_{0, m}^{\prime}\right),
$$

the projective limit being taken with respect to the restriction maps.

It is known that the reciprocity law map induces isomorphisms

$$
\operatorname{Gal}\left(M_{0, m} / k\right) \cong\left(J_{k} / k^{\times} B C\right)^{(p)} \text { and } \operatorname{Gal}\left(L_{0, m}^{\prime} / k\right) \cong\left(J_{k} / k^{\times} A C\right)^{(p)} .
$$

For this, see Sumida [13, pp. 692-693]. Therefore, we obtain a canonical isomorphism

$$
\operatorname{Gal}\left(M_{0, m} / L_{0, m}^{\prime}\right) \cong\left(k^{\times} A C / k^{\times} B C\right)^{(p)} \cong\left(A /\left(A \cap\left(k^{\times} B C\right)\right)\right)^{(p)} .
$$

We easily see that

$$
A \cap\left(k^{\times} B C\right)=\left(W^{(m)} \cap\left(E_{0}^{\prime} \mathcal{U}_{0}^{p^{m}}\right)\right) \times \prod_{\mathfrak{q} \nmid p}\{1\} .
$$

Here, we are regarding $E_{0}^{\prime}$ as a subgroup of $\prod_{\mathfrak{p} \mid p} k_{0, \mathfrak{p}}^{\times}$in the natural way. Hence, we have

$$
\operatorname{Gal}\left(M_{0, m} / L_{0, m}^{\prime}\right) \cong\left(W^{(m)} /\left(W^{(m)} \cap\left(E_{0}^{\prime} \mathcal{U}_{0}^{p^{m}}\right)\right)\right)^{(p)} .
$$

From this and (7), we obtain

$$
\operatorname{Gal}\left(M_{0} / L_{0}^{\prime}\right) \cong \mathcal{W}_{0} /\left(\mathcal{W}_{0} \cap \mathcal{E}_{0}^{\prime}\right)
$$

by an elementary but tedious argument on the topology of $\widehat{k_{0}^{\times}}=\left(\prod_{\mathfrak{p} \mid p} k_{0, \mathfrak{p}}^{\times}\right)^{(p)}$, which we leave to the reader. 


\subsection{Proof of Lemmas 1 and 2 .}

Proof of Lemma 1 (and the formula (2)). Let $B_{n}$ be the subgroup of $A_{n}$ consisting of classes which contain a product of prime ideals of $k_{n}$ over $p$, and let $B_{\infty}$ be the projective limit of $B_{n}$ with respect to the relative norms.

From class field theory, we see that $\mathcal{I}^{\prime} / \mathcal{I}$ is canonically isomorphic to $B_{\infty}$. Let $D(\subseteq \Delta)$ be the decomposition group of $p$ at $k$. Then, we have a natural surjection

$$
\mathbf{Z}_{p}[\Delta / D] \longrightarrow B_{\infty} \cong \mathcal{I}^{\prime} / \mathcal{I}
$$

over $\mathbf{Z}_{p}[\Delta]$. We see that $\mathbf{Z}_{p}[\Delta / D]\left(\Psi^{*}\right)=\{0\}$ or $O$ according as $\psi^{*}(p) \neq 1$ or $\psi^{*}(p)=1$. Let $\psi^{*}(p) \neq 1$. Then, from the above surjection, we see that $\mathcal{I}^{\prime}\left(\Psi^{*}\right)=\mathcal{I}\left(\Psi^{*}\right)$ (from which (2) follows). Hence, the assertion of Lemma 1 follows from (3) and (4) in this case.

Let $\psi^{*}(p)=1$. We have the following exact sequence of $\mathbf{Z}_{p}[\Delta]$-modules.

$$
\{0\} \longrightarrow \mathcal{U}_{0} \longrightarrow\left(\prod_{\mathfrak{p} \mid p} k_{0, \mathfrak{p}}^{\times}\right)^{(p)} \longrightarrow \mathbf{Z}_{p}[\Delta / D] \longrightarrow\{0\} .
$$

As $\psi^{*}(p)=1$, we see from (5) that

$$
\left(\mathcal{W}_{0} \cap \mathcal{U}_{0}\right)\left(\Psi^{*}\right)=\mathcal{V}_{0}\left(\Psi^{*}\right)=\{0\}
$$

Therefore, by the above exact sequence, we see that the $O$-module $\mathcal{W}_{0}\left(\Psi^{*}\right)$ is free of rank one (or $\left.\mathcal{W}_{0}\left(\Psi^{*}\right)=\{0\}\right)$. Hence, $\mathcal{W}_{\infty}\left(\Psi^{*}\right)$ is cyclic over $\Lambda$ by Lemma 3 and Nakayama's lemma (cf. [14, Lemma 13.16]). By this and Proposition $2, \mathcal{I}^{\prime}\left(\Psi^{*}\right)$ is cyclic over $\Lambda$. Then, we obtain $\mathcal{I}^{\prime}\left(\Psi^{*}\right) \cong \Lambda$ since $\mathcal{I} \subseteq \mathcal{I}^{\prime}$ and $\mathcal{I}\left(\Psi^{*}\right) \cong \Lambda$ by (3) and (4).

Proof of Lemma 2. It is known (cf. [6, Proposition 3]) that

$$
M\left(\Psi^{*}\right)=N\left(\Psi^{*}\right) L\left(\Psi^{*}\right) .
$$

Let $\psi^{*}(p) \neq 1$. Then, $N^{\prime}\left(\Psi^{*}\right)=N\left(\Psi^{*}\right)$ and $L^{\prime}\left(\Psi^{*}\right)=L\left(\Psi^{*}\right)$ by (1) and (2). Hence, the assertion follows from the above in this case. Let $\psi^{*}(p)=1$. Then, by Lemma $1, \mathcal{I}^{\prime}\left(\Psi^{*}\right) \cong \Lambda$. On the other hand, $\mathcal{Z}^{\prime}\left(\Psi^{*}\right)$ is finitely generated and torsion over $\Lambda$ by $[\mathbf{1 0}$, Theorems 5,14$]$. Therefore, we obtain $\mathcal{Z}^{\prime}\left(\Psi^{*}\right) \cap \mathcal{I}^{\prime}\left(\Psi^{*}\right)=\{0\}$, and hence $M\left(\Psi^{*}\right)=N^{\prime}\left(\Psi^{*}\right) L^{\prime}\left(\Psi^{*}\right)$.

\section{References}

[1] A. Brumer, On units of algebraic number fields, Mathematika, 14 (1967), 121-124, MR 36 \#3746, Zbl 0171.01105.

[2] B. Ferrero and L. Washington, The Iwasawa invariant $\mu_{p}$ vanishes for abelian number fields, Ann. Math., 109 (1979), 377-395, MR 81a:12005, Zbl 0443.12001. 
[3] R. Gillard, Unités cyclotomiques, unités semi-locales et $\mathbf{Z}_{\ell}$-extensions II, Ann. Inst. Fourier, 29 (1979), 1-15, MR 81e:12005b, Zbl 0403.12006.

[4] R. Greenberg, On the Iwasawa invariants of totally real number fields, Amer. J. Math., 98 (1976), 263-284, MR 53 \#5529, Zbl 0334.12013.

[5] H. Ichimura, On power integral bases of unramified cyclic extensions of prime degree, J. Algebra, 235 (2001), 104-112, MR 2001m:11199, Zbl 0972.11101.

[6] _ On a power integral bases problem over cyclotomic $\mathbf{Z}_{p}$-extensions, J. Algebra, 234 (2000), 90-100, CMP 1799479.

[7] _ On a quotient of the unramified Iwasawa module over an abelian number field, J. Number Theory, 88 (2001), 175-190, MR 2002b:11155, Zbl 0972.11104.

[8] real number field, to appear in Acta Arith.

[9] H. Ichimura and H. Sumida, On the Iwasawa invariants of certain real abelian fields II, International J. Math., 7 (1996), 721-744, MR 98e:11128c, Zbl 0881.11075.

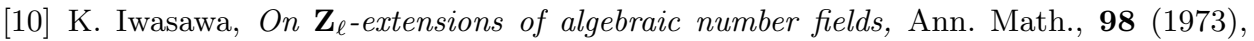
246-326, MR 50 \#2120, Zbl 0285.12008.

[11] J. Kraft and R. Schoof, Computing Iwasawa modules of real quadratic fields, Compositio Math., 97 (1995), 135-155, MR 97g:11120, Zbl 0840.11043.

[12] M. Kurihara, The Iwasawa $\lambda$-invariants of real abelian fields and the cyclotomic elements, Tokyo J. Math., 22 (1999), 259-277, MR 2001a:11182, Zbl 0941.11040.

[13] H. Sumida, Greenberg's conjecture and the Iwasawa polynomial, J. Math. Soc. Japan, 49 (1997), 689-711, MR 98h:11137, Zbl 0907.11038.

[14] L. Washington, Introduction to Cyclotomic Fields (2-nd edition), Springer, New York, 1996, MR 97h:11130, Zbl 0966.11047.

Received November 16, 2000 and revised November 28, 2001. The author was partially supported by Grant-in-Aid for Scientific Research (C), (No. 13640036), the Ministry of Education, Science, Sports and Culture of Japan.

Department of Mathematics

Yokohama City University

22-2, Seto, Kanazawa-Ku, Yokohama, 236-0027

JAPAN

E-mail address: ichimura@yokohama-cu.ac.jp 